\title{
UNBOUNDED DISJOINTNESS PRESERVING LINEAR FUNCTIONALS
}

\author{
LAWRENCE G. BROWN AND NGAI-CHING WONG
}

\begin{abstract}
Let $X$ be a locally compact Hausdorff space and $C_{0}(X)$ the Banach space of continuous functions on $X$ vanishing at infinity. In this paper, we shall study unbounded disjointness preserving linear functionals on $C_{0}(X)$. They arise from prime ideals of $C_{0}(X)$, and we translate it into the cozero set ideal setting. In particular, every unbounded disjointness preserving linear functional of $c_{0}$ can be constructed explicitly through an ultrafilter on $\mathbb{N}$ complementary to a cozero set ideal. This ultrafilter method can be extended to produce many, but in general not all, such functionals on $C_{0}(X)$ for arbitrary $X$. We also make some remarks where $C_{0}(X)$ is replaced by a non-commutative $\mathrm{C}^{*}$-algebra.
\end{abstract}

\section{INTRODUCTION.}

Let $X$ be a locally compact Hausdorff space and let $C_{0}(X)$ be the Banach algebra of continuous (real or complex) functions defined on $X$ vanishing at infinity and equipped with the supremum norm. It is well-known that every maximal ideal of $C_{0}(X)$ is the kernel of a multiplicative linear functional. Although this is not so for prime ideals, if $\varphi$ is a linear functional on $C_{0}(X)$ with kernel containing a prime ideal $I$ then $\varphi$ does preserve some algebraic properties of $C_{0}(X)$. In fact, $\varphi$ is disjointness preserving; namely,

$$
f g=0 \Longrightarrow \varphi(f) \varphi(g)=0, \quad f, g \in C_{0}(X) .
$$

Let coz $f=\{x \in X: f(x) \neq 0\}$ be the cozero set of $f$ for each $f$ in $C_{0}(X)$. Then $\varphi$ sends functions with disjoint cozero sets to disjoint scalars (i.e. one of them is zero). Indeed, since $I$ is prime, one of $f$ and $g$ belongs to $I$. As $\left.\varphi\right|_{I}=0$, one of $\varphi(f)$

Date: May 29, 2002; a version submitted to Monatshefte für Mathematik.

2000 Mathematics Subject Classification. 46J10, 46H40, 46L05.

Key words and phrases. unbounded disjointness preserving linear functionals, cozero set ideals, ultrafilters, $\mathrm{C}^{*}$-algebras.

Correspondence author: Ngai-Ching Wong, Department of Applied Mathematics, National Sun Yat-sen University, Kaohsiung, Taiwan 80424, ROC. E-mail: wong@math.nsysu.edu.tw . 
and $\varphi(g)$ must be zero. If $I$ is contained in a (necessarily unique) maximal ideal $M$, then the cardinality of the set of unbounded disjointness preserving linear functionals vanishing on $I$ modulo scalar multiples is at least $\operatorname{dim}(M / I)$. In this paper, we shall give a closer look at such unbounded linear functionals. To enumerate all possibilities between $I$ and $M$, we use the concept of prime cozero set ideals $\mathfrak{I}$.

Recently there is an increasing interest in the study of disjointness preserving linear operators (see e.g. $[5,1,13,11,8,12,2,3]$ ) between various spaces. A linear operator $T$ from $C_{0}(X)$ into $C_{0}(Y)$ is said to be disjointness preserving (or separating) if $f \cdot g=0$ in $C_{0}(X)$ implies $T f \cdot T g=0$ in $C_{0}(Y)$. In this case, the linear functional $\delta_{y} \circ T$ of $C_{0}(X)$ is disjointness preserving for every point $y$ in $Y$, where $\delta_{y} \circ T(f)=T f(y)$, $\forall f \in C_{0}(X)$. In particular, $T$ is bounded if and only if all such disjointness preserving linear functionals are bounded. Such a functional is bounded exactly when it is a scalar multiple of a point mass. In this case, $\delta_{y} \circ T=h(y) \delta_{\varphi(y)}$ for some scalar $h(y)$ and point $\varphi(y)$ in $Y$. Consequently, a disjointness preserving linear operator $T$ is bounded if and only if it is a weighted decomposition operator $T f=h \cdot f \circ \varphi$.

A better understanding of unbounded disjointness preserving linear functionals will benefit those working on disjointness preserving operators, especially on the problem of auto continuity of such operators. In [11], it is shown that there is always an unbounded disjointness preserving linear operator from $C_{0}(X)$ onto an infinite dimensional subspace of $C_{0}(Y)$ provided $X$ and $Y$ are infinite sets. In particular, the existence of disjointness preserving linear functionals of $C_{0}(X)$ is established (see also $[1,7])$.

In other contexts, for example in Gillman and Jerison [9], cozero set ideals have been used to good effect. In this paper, we show that the study of the appropriate sort of cozero set ideals provides a systematic way to study disjointness preserving linear functionals. In case $X=\mathbb{N}$, we show that all such functionals arise from ultrafilters on $\mathbb{N}$, which are complementary to prime cozero set ideals. This also gives us an easy way to construct many such functionals on $C_{0}(X)$ for arbitrary $X$. However, we 
provide examples to show that the ultrafilter method cannot give us all of them in general.

In the final section, we give preliminary consideration to the case where $C_{0}(X)$ is replaced by a general $\mathrm{C}^{*}$-algebra.

\section{Cozero SET IDEALS AND DISJOINTNESS PRESERVING LINEAR FUNCTIONALS}

Throughout the rest of this paper, $X$ denotes a locally compact Hausdorff space and $\mathfrak{M}$ denotes the family of all $\sigma$-compact open subsets of $X$. In other words, an open subset $U$ of $X$ is in $\mathfrak{M}$ if and only if it is the cozero set of an $f$ in $C_{0}(X)$. For $U$ in $\mathfrak{M}$, we identify $C_{0}(U)$ with $\left\{f \in C_{0}(X): \operatorname{coz}(f) \subseteq U\right\}$.

Definition 2.1. A nonempty subfamily $\mathfrak{I}$ of $\mathfrak{M}$ is said to be a cozero set ideal (on $X)$ if it satisfies the following conditions.

$(\mathrm{COZ} 0) \mathfrak{I} \neq \mathfrak{M}$.

(COZ1) If $U \in \mathfrak{I}, V \in \mathfrak{M}$ and $V \subseteq U$, then $V \in \mathfrak{I}$.

(COZ2) If $U \in \mathfrak{I}$ and $V \in \mathfrak{I}$, then $U \cup V \in \mathfrak{I}$.

A cozero set ideal $\mathfrak{I}$ is said to be prime if it satisfies

(COZ3) If $U, V \in \mathfrak{M}$ and $U \cap V=\emptyset$, then $U \in \mathfrak{I}$ or $V \in \mathfrak{I}$.

Lemma 2.2. A cozero set ideal $\mathfrak{I}$ is prime if and only if it satisfies

$\left(\mathbf{C O Z}^{\prime}\right)$ If $U, V \in \mathfrak{M}$ and $U \cap V \in \mathfrak{I}$, then $U \in \mathfrak{I}$ or $V \in \mathfrak{I}$.

Proof. It is plain that (COZ3') implies (COZ3). Conversely, suppose $U, V \in \mathfrak{M}$ such that $U \cap V \in \mathfrak{I}$. Consider the sets $A=U \backslash V$ and $B=V \backslash U$. These are disjoint, relatively closed subsets of the normal space $U \cup V$. So there are disjoint sets $W_{1}$ and $W_{2}$ in $\mathfrak{M}$ with $A \subseteq W_{1}$ and $B \subseteq W_{2}$. One of $W_{1}$ and $W_{2}$ must be in $\mathfrak{I}$. If, say, $W_{1}$ is in $\mathfrak{I}$ then $U \subseteq W_{1} \cup(U \cap V)$. This implies $U \in \mathfrak{I}$.

Lemma 2.3. Let $\mathfrak{C}$ be a subfamily of $\mathfrak{M}$ closed under finite intersections and $\emptyset \notin \mathfrak{C}$. Then any cozero set ideal $\mathfrak{I}$ maximal with respect to not containing any member of $\mathfrak{C}$ is prime. 
Proof. Suppose $V, W \notin \mathfrak{I}$. We want to see that $V \cap W \notin \mathfrak{I}$. Indeed, there are $U_{1}, U_{2}$ in $\mathfrak{C}$ and $A_{1}, A_{2}$ in $\mathfrak{I}$ such that $U_{1} \subseteq V \cup A_{1}$ and $U_{2} \subseteq W \cup A_{2}$ by the maximality of I. Therefore, $U_{1} \cap U_{2} \subseteq(V \cap W) \cup A_{1} \cup A_{2}$. As a result, $V \cap W$ cannot be in $\mathfrak{I}$.

Let $\mathfrak{I}$ be a cozero set ideal. Set

$$
P(\mathfrak{I})=\left\{f \in C_{0}(X): \operatorname{coz}(f) \in \mathfrak{I}\right\} .
$$

Then $P(\mathfrak{I})$ is a subspace of $C_{0}(X)$, even an ideal by $(\mathbf{C O Z} 1)$. And $P(\mathfrak{I}) \neq C_{0}(X)$ by $(\mathbf{C O Z} 0)$. So there exists a non-zero linear functional $\varphi$ on $C_{0}(X)$ such that $\left.\varphi\right|_{P(\mathfrak{I})}=0$. Moreover, $\mathfrak{I}$ is prime if and only if $P(\mathfrak{I})$ is a prime ideal of $C_{0}(X)$ by $\left(\mathbf{C O Z} 3^{\prime}\right)$. In this case, any linear functional $\varphi$ vanishing on $P(\mathfrak{I})$ is disjointness preserving by (COZ3).

On the other hand, for every nonzero linear functional $\varphi$ on $C_{0}(X)$, the kernel ideal of $\varphi$,

$$
\mathfrak{I}_{\varphi}=\left\{U \in \mathfrak{M}:\left.\varphi\right|_{C_{0}(U)}=0\right\},
$$

is a cozero set ideal, and $\varphi$ vanishes on $P\left(\mathfrak{I}_{\varphi}\right) . \mathfrak{I}_{\varphi}$ is prime if and only if $\varphi$ is disjointness preserving. However, if we start with a prime cozero set ideal $\mathfrak{I}$ and let $\varphi$ be any (disjointness preserving) linear functional vanishing on $P(\mathfrak{I})$ then we might have $\mathfrak{I}_{\varphi} \supsetneq \mathfrak{I}$.

For $x$ in $X$, set

$$
\mathfrak{I}_{x}=\{U \in \mathfrak{M}: x \notin U\}
$$

and

$$
M_{x}=\left\{f \in C_{0}(X): f(x)=0\right\} .
$$

Clearly, $\mathfrak{I}_{x}$ is a maximal cozero set ideal of $\mathfrak{M}$ and $M_{x}$ is a maximal ideal of $C_{0}(X)$. Moreover, $P\left(\mathfrak{I}_{x}\right)=M_{x}$.

Proposition 2.4. Let $\mathfrak{I}$ be a prime cozero set ideal.

(i) For all but at most one point $x$ in $X$, some neighborhood of $x$ is in $\mathfrak{I}$.

(ii) For $x$ in $X$, the following are equivalent.

(a) No neighborhood of $x$ is in $\mathfrak{I}$.

(b) $\mathfrak{I} \subseteq \mathfrak{I}_{x}$.

(c) $P(\mathfrak{I}) \subseteq M_{x}$. 
(d) $U \in \mathfrak{M}$ and $x \notin \bar{U}$ implies $U \in \mathfrak{I}$.

Proof. We verify only the implication from (d) to (a) in (ii). Suppose $V$ in $\mathfrak{I}$ is a neighborhood of $x$. Let $K$ be a compact $G_{\delta}$ neighborhood of $x$ such that $K \subseteq V$. For arbitrary $U$ in $\mathfrak{M}, U \backslash K$ is in $\mathfrak{I}$ by $(\mathrm{d})$. Since $U \subseteq(U \backslash K) \cup V$, we conclude that $U$ is in $\mathfrak{I}$, in contradiction with $(\mathbf{C O Z} 0)$.

In case $\mathfrak{I}=\mathfrak{I}_{x}$, every nonzero linear functional $\varphi$ vanishing on $P(\mathfrak{I})$ is a multiple of the point mass $\delta_{x}$. In this case, $P(\mathfrak{I})=M_{x}$ and $\mathfrak{I}=\mathfrak{I}_{\varphi}$. If $\mathfrak{I}$ is a proper subset of some $\mathfrak{I}_{x}$, then we have a $U$ in $\mathfrak{M}$ such that $x \notin U$ and $U \notin \mathfrak{I}$. By Proposition 2.4(ii), $x \in \bar{U}$. For any $f$ in $C_{0}(X)$ with $\operatorname{coz} f=U$, we can choose a disjointness preserving linear functional $\varphi$ on $C_{0}(X)$ so that $\varphi$ vanishes on $P(\mathfrak{I})$ and $\varphi(f) \neq 0$. Then, $\varphi$ is unbounded. If $x$ does not exist as above, $\mathfrak{I}$ contains $\{U \in \mathfrak{M}: \bar{U}$ is compact $\}$ and $P(\mathfrak{I})$ contains all continuous functions with compact support. In this case, any nonzero $\varphi$ vanishing on $P(\mathfrak{I})$ is unbounded and disjointness preserving, and we say that $\mathfrak{I}$ is associated to $\infty$.

The following proposition follows from a result of Jarosz [11] (see also [1] and [7]), but we present here a more direct argument.

Proposition 2.5. For every locally compact Hausdorff space $X$ of infinite cardinality, $C_{0}(X)$ admits an unbounded disjointness preserving linear functional $\varphi$.

Proof. First, assume we can choose a point $x$ in $X$ and a set $U$ in $\mathfrak{M}$ such that $x \in \bar{U} \backslash U$. Then let $\mathfrak{I}^{\prime}=\{V \in \mathfrak{M}: x \notin \bar{V}\}$. Clearly, $\mathfrak{I}^{\prime}$ is a cozero set ideal and $U \notin \mathfrak{I}^{\prime}$. Let $\mathfrak{I}$ be a cozero set ideal maximal with respect to $U \notin \mathfrak{I}$ and $\mathfrak{I} \supseteq \mathfrak{I}^{\prime}$. By Lemma $2.3, \mathfrak{I}$ is a prime cozero set ideal. Choose $f$ in $C_{0}(X)$ with coz $f=U$ and choose a nonzero $\varphi$ vanishing on $P(\mathfrak{I})$ so that $\varphi(f) \neq 0$. Then $\varphi$ is an unbounded disjointness preserving linear functional associated to $x$.

On the other hand, if there is a $U$ in $\mathfrak{M}$ such that $\bar{U}$ is not compact, then let $\mathfrak{I}$ be a cozero set ideal containing $\{V \in \mathfrak{M}: \bar{V}$ is compact $\}$ and maximal with respect to $U \notin \mathfrak{I}$. By Lemma 2.3 again, $\mathfrak{I}$ is prime. Then in a similar manner as above, we will have an unbounded disjointness preserving linear functional $\varphi$ associated to $\infty$. 
If the second of these cases does not occur, then every $\sigma$-compact subset of $X$ has compact closure. Hence, some point $x$ is a cluster point of a countably infinite set $\left\{x_{n}: n \in \mathbb{N}\right\}$. Let $V_{n}$ be disjoint neighborhoods of $x_{n}$ and $f_{n}$ in $C_{0}(X)$ with $\operatorname{coz}_{n} \subseteq V_{n}$ and $f_{n}\left(x_{n}\right)=\left\|f_{n}\right\|=1$ for all $n=1,2, \ldots$. Let $f=\sum_{n=1}^{\infty} 1 / 2^{n} f_{n}$ in $C_{0}(X)$. Note that $x \in \bar{U} \backslash U$, where $U=\operatorname{coz} f$. Thus the first case happens.

Remarks 2.6. (1) The second case in the proof of Proposition 2.5 is not possible if $X$ is compact. Even for some non-compact space $X$, the closure of every $\sigma$-compact subset is compact; e.g. $X=[0, \beta)=\{0 \leq \alpha<\beta\}$ in the order topology, where $\beta$ is the first uncountable ordinal number. Also some nonisolated points $x$ may be impossible. It may be that $U \in \mathfrak{M}$ and $x \notin U$ imply $x \notin \bar{U} ;$ e.g. $x=\beta$ in $X=[0, \beta]$.

(2) In a letter to the authors, Z. Lipecki indicated that a similar result holds for $F$-lattices, i.e., a complete metrizable topological linear lattice. Recall that a linear functional $f$ of a linear lattice is said to be disjointness preserving if $|x| \wedge|y|=0$ implies $f(x) f(y)=0$. Using [4, Theorem 6], one can establish the existence of an unbounded disjointness preserving linear functional of any infinite dimensional F-lattice. It thus gives yet another proof of Proposition 2.5 .

Recall that an ultrafilter (see e.g. $[10$, p. 6]) $\mathfrak{F}$ on the index set $\mathbb{N}$ is a family of subsets of $\mathbb{N}$ satisfying the following conditions.

(UF0) $\emptyset \notin \mathfrak{F}$.

(UF1) $A \in \mathfrak{F}, B \subseteq \mathbb{N}$ and $A \subseteq B$ implies $B \in \mathfrak{F}$.

(UF2) $A, B \in \mathfrak{F}$ implies $A \cap B \in \mathfrak{F}$.

(UF3) For each subset $A$ of $\mathbb{N}$, either $A \in \mathfrak{F}$ or its complement $A^{\prime} \in \mathfrak{F}$.

We call $\mathfrak{F}$ a free ultrafilter if the intersection of all members of $\mathfrak{F}$ is empty. In this case, $\mathfrak{F}$ contains no finite subset of $\mathbb{N}$. 
One can find a proof of the following lemma in [11], or makes up another using [4, Lemma 2.7]. However, we present here an apparently more direct and easier argument.

Lemma 2.7. Let $\mathfrak{F}$ be any free ultrafilter on $\mathbb{N}$. Then the algebraic dimension of the quotient space

$$
\ell_{\infty} /\left\{y \in \ell_{\infty}: y \text { vanishes on an element of } \mathfrak{F}\right\}
$$

is at least the continuum.

Proof. First we note that the transcendence dimension of the real field $\mathbb{R}$ over the rational field $\mathbb{Q}$ is the continuum. Consider the points $f_{\lambda}=\left(1, \lambda, \lambda^{2}, \ldots, \lambda^{n}, \ldots\right)$ in $\ell_{\infty}$ where $\lambda$ runs through a transcendence basis of $\mathbb{R}$ over $\mathbb{Q}$ and $|\lambda|<1$ for all $\lambda$. For each $n$-tuple $k=\left(k_{1}, \ldots, k_{n}\right)$ of distinct non-negative integers, the determinant $D_{k}\left(\lambda_{1}, \ldots, \lambda_{n}\right)=\operatorname{det}\left(\lambda_{i}^{k_{j}}\right)$ is non-zero if $\lambda_{1}, \ldots, \lambda_{n}$ are distinct elements of the basis. Consequently, the vectors $\left(\lambda_{i}^{k_{1}}, \ldots, \lambda_{i}^{k_{n}}\right)$ are linearly independent, and hence no nontrivial linear combination of $f_{\lambda_{1}}, \ldots, f_{\lambda_{n}}$ has $n$ coordinates equal to 0 . Therefore, no such linear combination vanishes on a member of $\mathfrak{F}$ since every such must contain infinitely many elements.

Lemma 2.8. If $\mathfrak{I}$ is a prime cozero set ideal and $\mathfrak{I} \neq \mathfrak{I}_{x}$ for all $x$ in $X$, then $C_{0}(X) / P(\mathfrak{I})$ has algebraic dimension at least the continuum.

Proof. Let $x$ in $X \cup\{\infty\}$ be associated to $\mathfrak{I}$ as in Proposition 2.4. Since $\mathfrak{I} \neq \mathfrak{I}_{x}$ (and $\mathfrak{I} \neq \mathfrak{I}_{\infty}=\mathfrak{M}$ by $\left.(\mathbf{C O Z} 0)\right)$, there is a $U$ in $\mathfrak{I}_{x} \backslash \mathfrak{I}$. We can write $U=\cup_{n=1}^{\infty} K_{n}$ where $K_{n}$ is compact and $K_{n}$ is contained in the interior $K_{n+1}^{o}$ of $K_{n+1}$. Set $K_{0}=\emptyset$. Then we can find $U_{n}$ in $\mathfrak{M}$ such that $U_{n} \subseteq K_{n+1}^{o} \backslash K_{n-1}$ and $K_{n} \subseteq U_{1} \cup \cdots \cup U_{n}$. Let $V=\cup_{n \text { even }} U_{n}$ and $W=\cup_{n}$ odd $U_{n}$. Since $U=V \cup W$, at least one of $V$ and $W$ is not in $\mathfrak{I}$. Let $V \notin \mathfrak{I}$, say. Note that $U_{n} \cap U_{m}=\emptyset$ if $|n-m| \geq 2$, and by Proposition 2.4(iid), $U_{n} \in \mathfrak{I}, \forall n=1,2, \ldots$ So we have $V=\cup_{n=1}^{\infty} V_{n}$, where $V_{n}$ 's are disjoint and non-empty, each $V_{n}$ is in $\mathfrak{I}$, and $V$ is not in $\mathfrak{I}$. Define a free ultrafilter $\mathfrak{F}$ on $\mathbb{N}$ by

$$
E \in \mathfrak{F} \quad \Leftrightarrow \quad \cup_{n \in E} V_{n} \notin \mathfrak{I} .
$$


Choose an $f$ in $C_{0}(X)$ with $\operatorname{coz} f=V$. For each bounded sequence $y=\left(y_{n}\right)$ in $\ell_{\infty}$, define $g_{y}$ by $\left.g_{y}\right|_{V_{n}}=\left.y_{n} f\right|_{V_{n}}$ for $n=1,2, \ldots$, and $\left.g_{y}\right|_{X \backslash V}=0$. Then $g_{y} \in C_{0}(X)$, $\operatorname{coz}_{y} \subseteq V$, and $g_{y} \in P(\mathfrak{I})$ if and only if $\left\{n \in \mathbb{N}: y_{n}=0\right\} \in \mathfrak{F}$. So

$$
\operatorname{dim}\left(C_{0}(X) / P(\mathfrak{I})\right) \geq \operatorname{dim}\left(\ell_{\infty} /\left\{y \in \ell_{\infty}: y \text { vanishes on an element of } \mathfrak{F}\right\}\right),
$$

which is at least the continuum by Lemma 2.7 .

If $X$ is second countable then $\mathfrak{M}$ has cardinality at most the continuum. Thus the following theorem applies.

Theorem 2.9. If $\mathfrak{M}$ has cardinality at most the continuum, then any prime cozero set ideal $\mathfrak{I}$ is the kernel ideal $\mathfrak{I}_{\varphi}$ of a disjointness preserving linear functional $\varphi$ of $C_{0}(X)$.

Proof. We may suppose $\mathfrak{I} \neq \mathfrak{I}_{x}$ for any $x$ in $X$, for otherwise we can set $\varphi$ to be the point mass $\delta_{x}$. We can index the elements of $\mathfrak{M} \backslash \mathfrak{I}$ as follows.

$$
\mathfrak{M} \backslash \mathfrak{I}=\left\{U_{\alpha}: 0 \leq \alpha<\beta\right\},
$$

where $\beta$ is an ordinal number and the cardinality of each $\gamma<\beta$ is less than the continuum. Then we can use transfinite recursion to find $f_{\alpha}$ in $C_{0}\left(U_{\alpha}\right)$ such that the image of the $f_{\alpha}$ 's in $C_{0}(X) / P(\mathfrak{I})$ are linearly independent. This can be done since by Lemma 2.8, $C_{0}\left(U_{\alpha}\right) / C_{0}\left(U_{\alpha}\right) \cap P(\mathfrak{I})$ has dimension at least the continuum. Consequently, we can find a linear functional $\varphi$ such that $\left.\varphi\right|_{P(\mathfrak{I})}=0$ and $\varphi\left(f_{\alpha}\right) \neq 0$ for all $\alpha$. Clearly, $\varphi$ is disjointness preserving with $\mathfrak{I}_{\varphi}=\mathfrak{I}$.

By the proof of the above theorem, for any prime cozero set ideal $\mathfrak{I}$ maximal with respect to not containing a particular $U$ in $\mathfrak{M}$, we have a disjointness preserving linear functional $\varphi$ such that $\mathfrak{I}_{\varphi}=\mathfrak{I}$. In fact, we have the more general

Proposition 2.10. Let $\mathfrak{C}$ be a subfamily of $\mathfrak{M}$ closed under finite intersections with $\emptyset \notin \mathfrak{C}$. If the cardinality of $\mathfrak{C}$ is at most the continuum then for any cozero set ideal $\mathfrak{I}$ maximal with respect to not containing any member of $\mathfrak{C}$, there is a disjointness preserving linear functional $\varphi$ of $C_{0}(X)$ such that $\mathfrak{I}_{\varphi}=\mathfrak{I}$. 
Proof. By Lemma 2.3, $\mathfrak{I}$ is prime. Write $\mathfrak{C}=\left\{U_{\alpha}: 0 \leq \alpha<\beta\right\}$, where $\beta$ is an ordinal number and the cardinality of each $\gamma<\beta$ is less than the continuum. By the same argument in the proof of Theorem 2.9, we will have a disjointness preserving linear functional $\varphi$ of $C_{0}(X)$ such that $\left.\varphi\right|_{P(\mathfrak{I})}=0$ but $\varphi\left(f_{\alpha}\right) \neq 0$ for a linear independent family of functions $f_{\alpha}$ each with cozero set contained in $U_{\alpha}$. Then $\mathfrak{I}_{\varphi}$ is a prime cozero set ideal containing $\mathfrak{I}$ but disjoint from $\mathfrak{C}$. By the maximality of $\mathfrak{I}$, we have $\mathfrak{I}_{\varphi}=\mathfrak{I}$.

\section{UNBOUNDED DISJOINTNESS PRESERVING LINEAR FUNCTIONALS ON $c_{0}$}

The Banach space $c_{0}$ can be identified with $C_{0}(\mathbb{N})$. Since every subset of $\mathbb{N}$ is a cozero set, it is easy to see that the prime cozero set ideals are precisely the maximal ideals of sets. In other words, there is a one-to-one correspondence between prime cozero set ideals $\mathfrak{I}$ and ultrafilters $\mathfrak{F}$, such that for any $U$ in $\mathfrak{M}$,

$$
U \in \mathfrak{I} \quad \Leftrightarrow \quad \mathbb{N} \backslash U \in \mathfrak{F}
$$

The principle ultrafilters correspond to the ideals $\mathfrak{I}_{n}$, for $n$ in $\mathbb{N}$, and thus to bounded disjointness preserving linear functionals. If $\mathfrak{F}$ is a free ultrafilter, the corresponding ideal $\mathfrak{I}$ is associated to the point $\infty$ of $\mathbb{N} \cup\{\infty\}$. Thus, any nonzero (necessarily disjointness preserving) linear functional $\varphi$ vanishing on $P(\mathfrak{I})$ is unbounded. Also, since $\mathfrak{I}$ is maximal, $\mathfrak{I}_{\varphi}=\mathfrak{I}$. So the following is a special case of the results of Section 2 .

Proposition 3.1. For each free ultrafilter $\mathfrak{F}$ on $\mathbb{N}$, let

$$
P^{\prime}(\mathfrak{F})=\left\{x \in c_{0}: x \text { vanishes on a element of } \mathfrak{F}\right\}
$$

Then the unbounded disjointness preserving linear functionals on $c_{0}$ are precisely the non-zero linear functionals vanishing on $P^{\prime}(\mathfrak{F})$ for some $\mathfrak{F}$. Also $\mathfrak{F}$ is uniquely determined by $\varphi$.

Corollary 3.2. For each sequence $x$ in $c_{0}$ which is not eventually null, there is an unbounded disjointness preserving linear functional $\varphi$ on $c_{0}$ such that $\varphi(x)=1$. 


\section{Unbounded DisJointness PRESERVING LiNEAR FUnCTIONALS ON $C_{0}(X)$}

Suppose $X$ is a locally compact Hausdorff space. If $X$ is a finite set then all linear functionals on $C_{0}(X)$ are automatically continuous. In this section, we always assume $X$ has infinite cardinality. It follows from Proposition 2.4(ii) that if $\varphi$ is an unbounded disjointness preserving linear functional on $C_{0}(X)$ then there is a non-isolated point $x$ in $X \cup\{\infty\}$ such that for all $f$ in $C_{0}(X)$,

$$
f \text { vanishes in a neighborhood of } x \text { in } X \Longrightarrow \varphi(f)=0 \text {. }
$$

We also note that $\varphi$ is unbounded if and only if $\varphi(f) \neq 0$ for some $f$ in $C_{0}(X)$ with $f(x)=0$.

Proposition 4.1. Let $x$ be an non-isolated point in $X$. Let $f$ in $C_{0}(X)$ be such that $f(x)=0$ but $f$ does not vanish in any neighborhood of $x$. Then there is an unbounded disjointness preserving linear functional $\varphi$ on $C_{0}(X)$ such that $\varphi(f)=1$.

Proof. This follows from the proof of Proposition 2.5.

In the rest of this section, we would like to see to what extent we can make use of the ultrafilter to give a more concrete construction of disjointness preserving linear functionals.

Definition 4.2. Let $X$ be a locally compact Hausdorff space. A closed subset $E$ of $X$ is called special if it is a disjoint union $E=\left\{e_{n}: n \in \mathbb{N}\right\} \cup E_{\infty}$, where the $e_{n}$ 's are distinct isolated points of $E$ and $E_{\infty}$ is the set of cluster points of the sequence $\left\{e_{n}\right\}$. In other words, $E$ is special if and only if there is a countable infinite subset $E_{0}$ of $E$ which is discrete in the relative topology such that $E=\overline{E_{0}}$. If $E_{0}$ is also closed, then $E_{\infty}=\emptyset$.

Lemma 4.3. Let $E$ be a special subset of $X$ and let $\mathfrak{P}_{E}$ be the set of prime cozero set ideals $\mathfrak{I}$ such that $\{U \in \mathfrak{M}: U \cap E=\emptyset\} \subseteq \mathfrak{I}$ and $\left\{U \in \mathfrak{M}: U \cap E_{\infty}=\emptyset\right\} \nsubseteq \mathfrak{I}$. Then there is a one-to-one correspondence between $\mathfrak{P}_{E}$ and the set of ultrafilters $\mathfrak{F}$ on $\mathbb{N}$ given by

$$
U \in \mathfrak{I} \quad \Leftrightarrow \quad\left\{n \in \mathbb{N}: e_{n} \notin U\right\} \in \mathfrak{F} .
$$


Proof. We first note that if $\mathfrak{I}$ is in $\mathfrak{P}_{E}$ and $U, V$ are in $\mathfrak{M}$ such that $U \cap E=V \cap E$, then

$$
U \in \mathfrak{I} \quad \Leftrightarrow \quad V \in \mathfrak{I} .
$$

In fact if, say, $V$ is in $\mathfrak{I}$, then $U \backslash V$ is a $\sigma$-compact set disjoint from $E$. Hence there is a $W$ in $\mathfrak{M}$ such that $W \cap E=\emptyset$ and $(U \backslash V) \subseteq W$. Thus $U \subseteq V \cup W$ and $W \in \mathfrak{I}$. Consequently, $U \in \mathfrak{I}$. Also note that the Tietze extension theorem implies that every cozero set of an element of $C_{0}(E)$ is the intersection with $E$ of the cozero set of a element of $C_{0}(X)$. In other words, the family of $\sigma$-compact open subsets of $E$ is $\mathfrak{M}_{E}=\{U \cap E: U \in \mathfrak{M}\}$.

Now we see that $\mathfrak{I}_{E}=\{U \cap E: U \in \mathfrak{I}\}$ is a prime cozero set ideal on $E$ and that $\mathfrak{I}=\left\{U \in \mathfrak{M}: U \cap E \in \mathfrak{I}_{E}\right\}$. For example, we prove $(\mathbf{C O Z} 1)$ for $\mathfrak{I}_{E}$. Let $U \cap E \subseteq V \cap E$ and $V \cap E \in \mathfrak{I}_{E}$ (so that $V \in \mathfrak{I}$ ). Note that $(U \cup V) \cap E=V \cap E$. Hence $U \cup V \in \mathfrak{I}$, whence $U \in \mathfrak{I}$ and $U \cap E \in \mathfrak{I}_{E}$.

The set $U_{0}=\left\{e_{n}: n \in \mathbb{N}\right\}$ is in $\mathfrak{M}_{E}$, and it is impossible that $U_{0} \in \mathfrak{I}_{E}$. Otherwise, $\left\{U \in \mathfrak{M}: U \cap E_{\infty}=\emptyset\right\}$ would be contained in $\mathfrak{I}$. Since $\mathfrak{I}_{E}$ is prime, this implies

$$
V \in \mathfrak{I}_{E} \quad \Leftrightarrow \quad V \cap U_{0} \in \mathfrak{I}_{E}, \quad \forall V \in \mathfrak{M}_{E},
$$

Thus $\left\{V \cap U_{0}: V \in \mathfrak{I}_{E}\right\}$ is a prime ideal of subsets of $U_{0}$; or equivalently $\mathfrak{F}$, as defined by (2), is an ultrafilter.

Thus we have a one-to-one map, satisfying (2), from $\mathfrak{P}_{E}$ into the set of ultrafilters. But it is easy to see that this map is onto. In other words, if an ultrafilter $\mathfrak{F}$ is given and $\mathfrak{I}$ is defined by $(2)$, then $\mathfrak{I}$ is in $\mathfrak{P}_{E}$.

Example-Remark 4.4. If $\mathfrak{I}$ is in $\mathfrak{P}_{E}$ and $\mathfrak{I}_{E}$ is as above, then $\mathfrak{I}$ is associated with a point $x$ in $X \cup\{\infty\}$ and $\mathfrak{I}_{E}$ is associated with a point $e$ in $E \cup\{\infty\}$. It is not hard to see that $x=e$. First note that if $x \notin E \cup\{\infty\}$, then there is a $U$ in $\mathfrak{M}$ such that $U_{0}=\left\{e_{n}: n \in \mathbb{N}\right\} \subseteq U$ and $x \notin \bar{U}$. Hence $U$ is in $\mathfrak{I}$ by Proposition 2.4, contradicting the fact proved above that $U_{0} \notin \mathfrak{I}_{E}$. Now the claim follows easily from Proposition 2.4 again. 
It is clear that the ultrafilter $\mathfrak{F}$ is principal if and only if $x=e=e_{n}$ for some $n$. Also, if this does not occur, then $\mathfrak{I} \subsetneq \mathfrak{I}_{x}$. Thus the free ultrafilters correspond exactly to the prime cozero set ideals in $\mathfrak{P}_{E}$ which are associated to unbounded disjointness linear functionals.

Now, the choice of a special set $E$ leads to "examples" of unbounded disjointness linear functionals. To get such an example, we choose a free ultrafilter on $\mathbb{N}$ and a linear functional $\varphi$ on $C_{0}(E) / P\left(\mathfrak{I}_{E}\right)$ such that $\left.\varphi\right|_{c_{0}} \neq 0$. (Here we identify $c_{0}$ with $\left\{f \in C_{0}(X):\left.f\right|_{E_{\infty}}=0\right\}$.) Although these choices cannot really be made explicitly, it seems that these examples are as explicit as one could reasonably hope, and that they are deservedly called examples. Note also that whenever $X$ has infinitely many points, there is an infinite sequence of mutually disjoint, non-empty, open subsets. Hence special sets exist.

If a subset $E$ exists which is homeomorphic to the Stone-Cech compactification $\beta \mathbb{N}$ of $\mathbb{N}$, then $E$ is special. So consider the case where $E=X=\beta \mathbb{N}$. Thus $C_{0}(X)$ can be identified with $\ell_{\infty}$. The above theory implies that if $\varphi$ is an unbounded disjointness preserving linear functional on $\ell_{\infty}$, and if $\left.\varphi\right|_{c_{0}} \neq 0$, then $\mathfrak{I}_{\varphi}$ is determined by an ultrafilter on $\mathbb{N}$, and also $\mathfrak{I}_{\varphi}$ is determined by $\left.\varphi\right|_{c_{0}}$. However, $\varphi$ itself is not determined by $\left.\varphi\right|_{c_{0}}$, since $\ell_{\infty} \neq c_{0}+P\left(\mathfrak{I}_{\varphi}\right)$.

Finally, we note that these examples were essentially given in Jarosz [11]. Our purpose was not just to establish the existence of such examples, but to analyze how they fit into the totality of disjointness preserving linear functionals on $C_{0}(X)$.

The set of all unbounded disjointness preserving linear functionals generated in this way is an infinite set. However, we will see with counter examples that not every disjointness preserving linear functional can be obtained in this way. In particular, there is an unbounded disjointness preserving linear functional $\varphi$ on $C[0,1]$ associated to 0 , a $G_{\delta}$ point, such that $\varphi$ does not arise from the ultrafilter method.

Lemma 4.5. The union of two special sets is again special. 
Proof. Suppose $E$ and $F$ are two special sets of a locally compact Hausdorff space $X$. Write $E=\left\{e_{n}: n=1,2, \ldots\right\} \cup E_{\infty}$ and $F=\left\{f_{m}: m=1,2, \ldots\right\} \cup F_{\infty}$. If some $e_{n}$ is not in $F_{\infty}$ then $e_{n}$ is isolated in $E \cup F$. If $e_{n}$ is in $F_{\infty}$, let $O$ be a neighborhood of $e_{n}$ in $X$ such that $O \cap E=\left\{e_{n}\right\}$, and let $A=\left\{f_{m}: m=1,2, \ldots\right\} \cap O$. Then $e_{n} \in \bar{A}$. Also since no element of $A$ is in $E_{\infty}$, each element of $A$ is isolated in $E \cup F$. Thus if $B$ is the set of isolated points of $E \cup F$, then each $e_{n}$ is in $\bar{B}$. Also each $f_{m}$ is in $\bar{B}$. So $\bar{B}=E \cup F$. Moreover, $B \cap\left(E_{\infty} \cup F_{\infty}\right)=\emptyset$. It follows that $B$ is countably infinite, and we can put $E \cup F$ in the special form by enumerating $B$.

Lemma 4.6. If $E$ is a special subset of $[0,1]$ then $E$ does not contain any neighborhood of 0 .

Proof. Let $O$ be an open neighborhood of 0 . Since $[0,1]$ has no isolated points, the set $O \backslash\left\{e_{n}: n \in \mathbb{N}\right\}$ is dense in $O$ (Baire Category Theorem). So if $O \subseteq E$, then $O \backslash\left\{e_{n}: n \in \mathbb{N}\right\} \subseteq E_{\infty}$, and hence $O \subseteq E_{\infty}$. But if $E_{\infty}$ contains a neighborhood of 0 , then 0 cannot be a cluster point of $\left\{e_{n}: n \in \mathbb{N}\right\}$, a contradiction.

The next result shows that there is an unbounded disjointness preserving linear functional on $C[0,1]$ which cannot be obtained by the ultrafilter method demonstrated in Example 4.4 .

Proposition 4.7. There is an unbounded disjointness preserving linear functional $\varphi$ on $C[0,1]$ such that $\mathfrak{I}_{\varphi}$ contains no set of the form $[0,1] \backslash E$ for a special set $E$. Hence $\mathfrak{I}_{\varphi}$ is not in $\mathfrak{P}_{E}$ for any special $E$.

Proof. We first note that every open subset of $[0,1]$ is $\sigma$-compact. Let $\mathfrak{B}=\{[0,1] \backslash E$ : $E$ is special $\}$ and $\mathfrak{I}^{\prime}=\{U \in \mathfrak{M}: U$ is open and $0 \notin \bar{U}\}$. Then $\mathfrak{B}$ and $\mathfrak{I}^{\prime}$ are subsets of $\mathfrak{M}, \mathfrak{B}$ is closed under finite intersections (Lemma 4.5 ), $\mathfrak{I}^{\prime}$ is closed under finite unions, and no element of $\mathfrak{I}^{\prime}$ includes an element of $\mathfrak{B}$ (Lemma 4.6). Now use Zorn's Lemma to find a set $\mathfrak{I}$ maximal with respect to: $\mathfrak{I}^{\prime} \subseteq \mathfrak{I} \subseteq \mathfrak{M}$, $\mathfrak{I}$ satisfies $(\mathbf{C O Z} 1)$ and (COZ2), and $\mathfrak{I} \cap \mathfrak{B}=\emptyset$. By Lemmas 2.3 and 4.6, $\mathfrak{I}$ satisfies $(\mathbf{C O Z} 3)$ and $(\mathbf{C O Z} 0)$. Clearly, $\mathfrak{I}$ is associated to 0 and $(0,1] \notin \mathfrak{I}$. This gives rise to a (necessarily unbounded) disjointness preserving linear functional $\varphi$ such that $\mathfrak{I}=\mathfrak{I}_{\varphi}$ by Proposition 2.10 . 
In the next example, we shall see that Example 4.4 gives rise to an unbounded disjointness preserving linear functional $\varphi$ satisfying: $\mathfrak{I}_{\varphi}$ is not maximal in $\mathfrak{I}_{x}$, but there is a $U$ in $\mathfrak{M} \backslash \mathfrak{I}_{\varphi}$ such that $\mathfrak{I}_{\varphi}$ is maximal with respect to the conditions that $\mathfrak{I}_{\varphi}$ satisfies $(\mathrm{COZ} 1)$ and $(\mathbf{C O Z} 2)$ and $U \notin \mathfrak{I}_{\varphi}$.

To get this we again take $X=[0,1]$. Let $C$ be the Cantor set. Choose a sequence $\left\{x_{n}\right\}$ of distinct points in $[0,1] \backslash C$ such that $C$ is the set of cluster points of $\left\{x_{n}\right\}$. Thus $E=\left\{x_{n}: n \in \mathbb{N}\right\} \cup C$ is special. Now let $\mathfrak{I}^{\prime}$ be the family of subsets $A$ of $\mathbb{N}$ with the property that there is an $\epsilon>0$ such that $C \cap(0, \epsilon)$ is disjoint from $\overline{\left\{x_{n}: n \in A\right\}}$. Then $\mathfrak{I}^{\prime}$ is closed under finite unions and subsets. Also, $\mathbb{N} \notin \mathfrak{I}^{\prime}$, but every finite subset of $\mathbb{N}$ is in $\mathfrak{I}^{\prime}$. So there is a free ultrafilter $\mathfrak{F}$ such that $\mathfrak{F} \cap \mathfrak{I}^{\prime}=\emptyset$. Using this ultrafilter, and $\left\{x_{n}\right\}$, we obtain an unbounded disjointness preserving linear functional $\varphi$ of $C[0,1]$ (necessarily associated to 0 ). Note that $U \in \mathfrak{I}_{\varphi}$ if and only if $\left\{n \in \mathbb{N}: x_{n} \notin U\right\}$ is in $\mathfrak{F}$. We will see in the following two lemmas that this $\varphi$ satisfies the properties stated in the previous paragraph.

Lemma 4.8. The kernel ideal $\mathfrak{I}_{\varphi}$ of $\varphi$ is not maximal in $\mathfrak{I}_{0}=\{U \in \mathfrak{M}: 0 \notin U\}$.

Proof. Let $U_{1}=[0,1] \backslash C$. Then $U_{1}$ is not in $\mathfrak{I}_{\varphi}$. So if $\mathfrak{I}_{\varphi}$ is maximal, there exists $V$ in $\mathfrak{I}_{\varphi}$ such that $(0,1]=U_{1} \cup V$. Let $A=\left\{n \in \mathbb{N}: x_{n} \notin V\right\}$. Since $A \in \mathfrak{F}$, $A \notin \mathfrak{I}^{\prime}$. Therefore, the set of cluster points of $\left\{x_{n}: n \in A\right\}$ is a subset $K$ of $C$ which intersects $(0, \epsilon)$, for all $\epsilon>0$. But $K \subseteq[0,1] \backslash V$, since $[0,1] \backslash V$ is closed. It follows that $K \backslash\{0\} \subseteq U_{1}$, a contradiction since $C \cap U_{1}=\emptyset$.

Lemma 4.9. $\mathfrak{I}_{\varphi}$ is maximal with respect to $(\mathbf{C O Z} 1),(\mathbf{C O Z} 2)$ and $U_{1} \notin \mathfrak{I}_{\varphi}$.

Proof. This follows from Lemma 4.3.

\section{Further CONSIDERATIONS}

Since $C_{0}(X)$ is a $\mathrm{C}^{*}$-algebra, it may be interesting to consider disjointness preserving maps between $\mathrm{C}^{*}$-algebras. There are two one-sided concepts of disjointness, which of course have identical theories. For elements $a, b$ of a $\mathrm{C}^{*}$-algebra $A$, we will interpret $a$ disjoint from $b$ to mean $a b^{*}=0$. (This is symmetric in $a$ and $b$ and is 
equivalent to orthogonality of the right support projections of $a$ and $b$. The right support projection of an element of $A$ is an open projection in $A^{* *}$.) A linear map $T: A \longrightarrow B$ will be called disjointness preserving if $a b^{*}=0$ implies $(T a)(T b)^{*}=0$. We content ourselves here with one theorem and one question about the case where $B$ is one-dimensional. Neither author has spent a lot of time thinking about disjointness preserving maps in the non-commutative case as of now.

Theorem 5.1. Any bounded disjointness preserving linear functional $\varphi$ on a complex $C^{*}$-algebra $A$ is a multiple of a ${ }^{*}$-homomorphism. In other words, $\varphi$ factors through the abelianization of $A$.

Proof. 1 . We reduce to the case $\varphi \geq 0$. We identify $\varphi$ with its extension to a weak* continuous functional on $A^{* *}$ and note that $A^{* *}$ is an von Neumann algebra. Then by [14, pp. 31-33] there is $u$ in $A^{* *}$ such that if $|\varphi|$ is defined by

$$
|\varphi|(a)=\varphi(u a), \quad \forall a \in A^{* *}
$$

then $|\varphi| \geq 0$ and

$$
\varphi(a)=|\varphi|\left(u^{*} a\right), \quad \forall a \in A^{* *}
$$

Since $u$ can be approximated in the weak* topology by elements of $A,|\varphi|$ is also disjointness preserving on $A$. And if $|\varphi|$ is a multiple of a $*$-homomorphism, then $\varphi$ is also, since then $\varphi(a)=|\varphi|\left(u^{*}\right)|\varphi|(a)$.

2. Changing notation, we assume $\varphi \geq 0$ and $\|\varphi\|=1$. Then $\varphi$ extends uniquely to a state $\tilde{\varphi}$ on $\tilde{A}$, where $\tilde{A}$ is the result of adjoining an identity to $A$. Let $\left(e_{i}\right)$ be an approximate identity of $A$, consisting of positive contractions. From the fact that $\|\tilde{\varphi}\|=\|\varphi\|$, we conclude $\tilde{\varphi}(b)=\lim _{i} \tilde{\varphi}\left(e_{i} b\right)=\lim _{i} \varphi\left(e_{i} b\right), \forall b \in \tilde{A}$. It follows that $\tilde{\varphi}$ also is disjointness preserving. Hence, we may change notation again and assume $A$ is unital.

3. The restriction of $\varphi$ to any unital commutative $\mathrm{C}^{*}$-algebra $B$ is a disjointness preserving state of $B$. Hence $\left.\varphi\right|_{B}$ is multiplicative. It follows that $\varphi\left(h^{2}\right)=\varphi(h)^{2}$ for all self-adjoint $h$ in $A$. By polarization and linearization, we see that $\varphi$ is a 
Jordan homomorphism. Then since

$$
\varphi\left(a^{*} a+a a^{*}\right)=\varphi\left(a^{*}\right) \varphi(a)+\varphi(a) \varphi\left(a^{*}\right)
$$

we see that $\varphi(a)=0$ implies $\varphi\left(a^{*} a\right)=\varphi\left(a a^{*}\right)=0$. Hence the kernel of $\varphi$ is a (necessarily maximal) two-sided ideal.

Remark 5.2. The above proof was designed with the hope of being relatively accessible to non-specialists in $\mathrm{C}^{*}$-algebras. A more conceptual proof uses closed left ideals and their associated closed projections and the "Urysohn Lemma" of C. A. Akemann [6]. This latter method can also be used for real $\mathrm{C}^{*}$-algebras, but the conclusion has to be modified in the real case. In the real case $\varphi$ factors through a quotient algebra of $\mathrm{A}$ isomorphic to either $\mathbb{R}, \mathbb{C}$, or $\mathbb{H}$, where $\mathbb{H}$ is the quaternion algebra. This implies that $\varphi$ factors through $A / I$, where $I$ is the closed two-sided ideal generated by $\left\{a h-h a: h^{*}=h\right\}$. This is the quotient universal for the property that every self-adjoint element is central.

Question 5.3. Does every unbounded disjointness preserving linear functional on a (complex) $C^{*}$-algebra $A$ factor through the abelianization of $A$ ?

It can be deduced from Theorem 5.1 that there is no non-zero disjointness preserving linear functional on a $\mathrm{C}^{*}$-algebra of the form $A \otimes M_{n}$ for $n \geq 2$. This is a positive evidence.

\section{REFERENCES}

[1] Y. A. Abramovich, Multiplicative representations of disjointness preserving operators, Indag. Math. 45 (1983), 265-279.

[2] Y. A. Abramovich and A. K. Kitover, A solution to a problem on invertible disjointness preserving operators, Proc. Amer. Math. Soc. 126 (1998), 1501-1505.

[3] Y. A. Abramovich and A. K. Kitover, Inverses of disjointness preserving operators, Mem. Amer. Math. Soc. 143 (2000), no. 679, 1-162.

[4] Y. A. Abramovich and Z. Lipecki, On ideals and sublattices in linear lattices and $F$-lattices, Math. Proc. Camb. Phil. Soc. 108 (1990), 79-87.

[5] Y. A. Abramovich, A. Veksler and V. Koldunov, On Operators preserving disjointness, Soviet Math. Dokl. 20 (1979), 1089-1093.

[6] C. A. Akemann, Left ideal structure of C*-algebras, J. Funct. Anal. 6 (1970), 305-317. 
[7] G. Dolinar, Stability of disjointness preserving mappings, Proc. Amer. Math. Soc. 130 (2001), $129-138$.

[8] J. J. Font and S. Hernandez, On separating maps between locally compact spaces, Arch. Math. (Bessel) 63 (1994), 158-165.

[9] L. Gillman and M. Jerison, Rings of Continuous Functions, Springer-Verlag, New York, 1976.

[10] K. Kuratowski, Topology, vol. 1, 2nd edition, Academic Press, 1972.

[11] K. Jarosz, Automatic continuity of separating linear isomorphisms, Can. Math. Bull. 33 (1990), 139-144.

[12] J. S. Jeang and N. C. Wong, Weighted composition operators of $C_{0}(X)$ 's, J. Math. Anal. Appl. 201 (1996), 981-993.

[13] B. de Pagter, A note on disjointness preserving operators, Proc. Amer. Math. Soc. 90 (1984), $543-549$.

[14] S. Sakai, $C^{*}$-algebras and $W^{*}$-algebras, Ergebnisse der Mathematik und ihrer Grenzgebiete, Band 60, Springer-Verlag, New York-Heidelberg, 1971.

Department of Mathematics, Purdue University, West Lafayette, IN 47907, USA

E-mail address: lgb@math.purdue.edu

Department of Applied Mathematics, National Sun Yat-sen University, Kaohsiung, 80424, TAIWAN, R.O.C.

E-mail address: wong@math.nsysu.edu.tw 